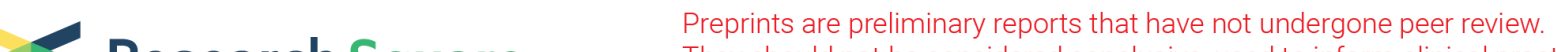 Research Square
or referenced by the media as validated information.
}

\section{Metastatic Ovariectomy is Beneficial for Survival in Ovarian Metastasis of Colorectal Carcinoma: A Single-center Experience in China}

\section{Min Jin}

Tongji Hospital of Tongji Medical College of Huazhong University of Science and Technology

\section{Lanqing Wang}

Tongji Hospital of Tongji Medical College of Huazhong University of Science and Technology

Lihua Luo

Tongji Hospital of Tongji Medical College of Huazhong University of Science and Technology Department of Radiology

\section{Dejun Zhang}

Tongji Hospital of Tongji Medical College of Huazhong University of Science and Technology

\section{Lei Zhao}

Tongji Hospital of Tongji Medical College of Huazhong University of Science and Technology

\section{Guojie Xu}

Tongji Hospital of Tongji Medical College of Huazhong University of Science and Technology Jianli Hu

Tongji Hospital of Tongji Medical College of Huazhong University of Science and Technology

Tao Zhang ( $\sim$ zhangtao_v123@163.com )

Huazhong University of Science and Technology https://orcid.org/0000-0001-8482-1954

\section{Research}

Keywords: Ovarian Metastasis, Colorectal Carcinoma, Ovariectomy, Chemotherapy

Posted Date: September 18th, 2020

DOI: https://doi.org/10.21203/rs.3.rs-72097/v1

License: (c) (i) This work is licensed under a Creative Commons Attribution 4.0 International License. Read Full License 


\section{Abstract}

\section{Background}

The benefit of ovarian resection in cases of ovarian metastases from colorectal carcinoma (CRC) is still uncertain. The purpose of this study is to explore the benefits of metastatic ovariectomy plus chemo over chemo alone in the therapy of ovarian metastasis of CRC and to ascertain prognostic factors.

\section{Methods}

A retrospective analysis of 54 female patients with ovarian metastasis of CRC was performed in a singlecenter between December 2009 and December 2017 in China. The subjects were divided into two subgroups: metastatic ovariectomy plus chemo subgroup and chemo alone subgroup. The clinicopathological features and overall survival (OS) were analyzed between the two subsets. Univariate and multivariate analysis were further performed to identify the risk factors for OS in these patients.

\section{Results}

The clinicopathological characteristics between the two subsets were similar, including 34 patients (63\%) with ovariectomy plus chemo and 20 patients (37\%) with chemo alone. In the total of 54 patients, the median survival time was 19.0 months ( $95 \%$ confidence interval, 5.0 to 60.0 months). The median survival time of metastatic tumor resection plus chemo subgroup was 27.9 months ( $95 \%$ confidence interval, 20.8 to 35.0 months), which was significantly better than that of simple chemo subgroup, 16.4 months ( $95 \%$ confidence interval, 11.8 to 21.0 months) $(P<0.01)$. When stratified analysis of the ovariectomy value in different time phases of ovarian metastasis, it indicated that ovariectomy plus chemo had superior survival benefits than chemo alone in CRC patients with metachronous metastasis (34.8 months vs. 18.4 months; $\mathrm{P}<0.05)$ but not synchronous metastasis $(17.2$ months vs.13.3months; $\mathrm{P}$ $=0.29)$.

\section{Conclusion}

Our results indicate that the removal of ovarian metastases of CRC can improve survival, especially in metachronous metastasis subjects. This study provides a support for ovariectomy of ovarian metastasis from CRC in clinical treatment for oncologist.

Running tittle

Treatment Strategy and Prognostic Analysis of Ovarian Metastasis of Colorectal Carcinoma

\section{Introduction}

Previous reports have shown that ovarian metastasis occurred in 2-8\% of female with metastatic CRC[13]. The ovary is one of the refuges of malignant cells. Because of its concealment, ovarian metastasis 
was infrequently to be early detected. In the past decade, with the continuous improvement of imaging, diagnosis and treatment of malignancies, the detection of CRC with ovarian metastases is frequently diagnosed over time[4]. However, the best treatment for CRC patients with ovarian metastasis is unclear and is still a controversial topic. There are no authoritative guidelines or high level of evidence-based medicine on the issue that whether the patients with ovarian metastasis from CRC should undergo metastatic ovariectomy.

In order to further understand the clinical role of ovariectomy, we focused on 56 female patients diagnosed with CRC with ovarian metastasis between 2009 to 2017 at the Cancer Center, Union Hospital, Huazhong University of Science and Technology in China. We conducted the survival analysis of ovariectomy plus chemo compared with chemo alone, and determined the clinicopathological features associated with OS improvement.

\section{Materials And Methods}

\section{Patients:}

We retrospectively reviewed the patients who were more than 18 years and pathologically diagnosed with CRC with ovarian metastasis from December 2009 to December 2017 at the Cancer Center, Wuhan Union Hospital in China. Upon the medical record database, a total of 1,984 patients were diagnosed with CRC. Among them, 78 (3.9\%) CRC female patients with ovarian metastasis were identified. After excluding neuroendocrine tumors, tumors besides CRC, the cases without treatment of chemo or surgery, 54 patients were identified in this research. Ovarian metastases of these CRC patients were confirmed with pathology of resected specimens or imaging evaluation of abdominal/ pelvic CT, MRI or ultrasonography. The institutional ethics review board in Wuhan Union Hospital approved this study. These subjects were divided into two subsets according their treatment: one is metastasectomy plus chemo and the other is chemo alone. The only metastasectomy group were relative small and were not included in this study, metastasectomy without chemotherapy was not recommended for these patients with advanced disease in the guidelines.

\section{Data gathering}

In this retrospective study, we reviewed patients' demographic and tumor features, pathology, treatment history, various hematological parameters and information on metastatic foci. OS is defined as the time from the date of pathological diagnosis of CRC to date of death or the last follow-up. The cut-off review date for OS was December 31, 2019. Synchronous metastasis refers to ovarian metastasis that occurs within 6 months after the diagnosis of CRC. Metachronous metastasis refers to ovarian metastasis that occurs 6 months after the diagnosis of CRC.

\section{Statistical analysis!}


All Statistical analysis was performed using SPSS software, version 17.0 (SPSS Inc., Chicago, IL, USA). A $P$ values $<0.05$ was considered a statistically significant difference. The kaplan-Meier method was used to calculate survival. Clinically relevant variables were analyzed univariately for their association with OS using the log-rank test. The independent prognostic significance of variables in terms of survival was determined in a multivariate analysis using the Cox proportional hazards regression model and the estimates are presented as hazard ratio (HR) with 95\% confidence interval (Cl).

\section{Results}

\section{Patient characteristics and treatment}

As shown in Table 1, the clinicopathological characteristics of ovarian metastasis of CRC between the two subgroups were balanced. Baseline data showed no statistically significant heterogeneity between the two groups. The median age was 45.3 ranging from 17.0 years to 75.0 years. The mean tumor size of ovarian metastasis was $10 \mathrm{~cm}$. But the median tumor size of cases with ovariectomy plus chemo was bigger than that of chemo alone cases (Table 1).

In the initial diagnosis, $65 \%$ cases had diatant metastasis in both group. The main pathology was adenocarcinoma in all cases with $25(94 \%)$ in ovariectomy plus chemo group and $15(97 \%)$ in chemo alone group. 65\% were poorly differentiated in both group. For the laterality, 20 (59\%) cases were unilateral in ovariectomy plus chemo group, while and 9 (45\%) cases in chemo alone group.

$43 \%$ of all cases suffered from synchronous ovarian metastasis in the initial diagnosis and the median ovarian metastasis onset of metachronous cases was 11 months (range, 1-48). The primary site of CRC are mainly colon $(93 \%, n=50)$. In addition to ovary, the common metastatic sits in these CRC subjects were peritoneum (48\%) \live (30\%) and lung (28\%). Of the 54 patients, $63 \%$ of the cases $(n=34)$ underwent ovarian metastasectomy plus chemo and $37 \%(n=20)$ underwent chemo alone. In the synchronous ovarian metastasis, $44 \%(n=15)$ of the patients underwent metastasectomy plus chemo, while $56 \%$ $(n=19)$ of the patients underwent metastasectomy plus chemo in the synchronous ovarian metastasis. There were $10(29 \%)$ cases used targeted therapy with bevacizumab in metastasectomy plus chemo group while $3(15 \%)$ cases in chemo only group.

\section{Survival outcome and prognostic factors}

In the entire cohort, the median OS of patients was estimated to be 19 months (95\% confidence interval [Cl], 5.5-60.6 months). As shown in Figure A, the median OS of metastasectomy plus chemo subgroup was 27.9 months $(95 \% \mathrm{Cl}, 20.8$ to 35.0 months), as compared with 16.4 months ( $95 \% \mathrm{Cl}, 11.8$ to 20.9 months) in chemo alone subgroup. Therefore, patients in metastasectomy plus chemo subgroup had a significant longer survival time than patients in chemo alone subgroup $(P<0.01)$. Additionally, 23 patients $(42.6 \%)$ of the participants had synchronously ovarian metastasis and 31 patients $(57.4 \%)$ had metachronous ovarian metastasis. The median OS of synchronously metastasis and metachronous metastasis were 15.6 months ( $95 \% \mathrm{Cl}, 11.8$ to 19.5 months) and 28.0 months ( $95 \% \mathrm{Cl}, 20.9$ to 35.0 
months), respectively (Figure B, Pख0.01). Moreover, it interestingly showed that metastasectomy plus chemo, relative to chemo alone, was significantly better for OS in patients with metachronous ovarian metastasis(34.8 months vs. 18.4 months; $P<0.05$ ) (Figure D), while in patients with synchronous ovarian metastasis, the OS trend between the two sub-groups did not get statistical significance(17.2 months vs. 13.3 months; $P=0.29$ ) (Figure $C$ ). Among the 13 patients who received bevacizumab treatment, the median OS was 30 months ( $95 \% \mathrm{Cl}, 25.5$ to 43.0 months) verus 17 months ( $95 \% \mathrm{Cl}, 15.5$ to 24.5 months) in 41 patients without bevacizumab treatment. According to the Response Evaluation Criteria in Solid Tumors, the response to bevacizumab combined therapy was partial remission (PR) in 5 cases (38.5\%), stable disease (SD) in 5 cases (38.5\%), and progressive disease (PD) in 3 cases (23.0\%).

Based-on univariate analysis, metastasectomy, metachronous ovarian metastasis, liver metastasis, lung

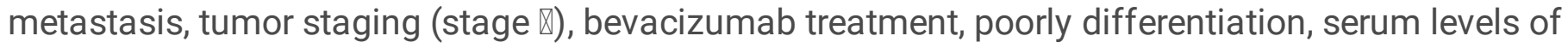
CA125 ( $>37 \mathrm{U} / \mathrm{mL}$ ) and CEA ( $>5 \mathrm{ng} / \mathrm{mL})$ were significant prognostic factors of OS. Previous studies showed that peritoneal metastasis was a risk factor for poor prognosis of CRC patients. The results of this study showed no significant difference, but $H R>1$ indicated that peritoneal metastasis had a certain adverse effect on the prognosis of patients. The insignificance of this data may be due to the fact that the majority cases had peritoneal metastases, and in chemo group, peritoneal metastasis may be underdiagnosed based on imaging. After adjustment for covariates on multivariate analysis, metastasectomy plus chemo (HR 0.4; 95\% Cl 0.20-0.79; $\mathrm{P}<0.01$ ), and metachronous ovarian metastasis (HR 0.46; $95 \% \mathrm{Cl}$ $0.22-0.95 ; P<0.05)$ were discovered as independent predictors of OS (Table 2). The level of serum CEA was marginally significant for predicting survival ( $\mathrm{HR} 2.04 ; 95 \% \mathrm{Cl} 0.90-4.59 ; \mathrm{P}=0.08$ ).

\section{Discussion}

CRC is one of the most frequent malignancies in China, and its morbidity and mortality are rising by years[5]. With the enormous advancements in treatment options, such as surgery, systemic chemo, local radiotherapy, ablation, targeted therapy and immunotherapy, the overall survival of advanced CRC increased to 3 years[6]. However, the survival rate is still actually unpleasant for those with metastasized disease. It has been shown that CRC patients with ovarian metastasis have a more poorer prognosis compared to other sites metastasis[7]. According to published data, the median OS of female CRC with ovarian metastasis was about 19 months[8]. It is speculated that the reason for the miserable prognosis of ovarian involvement is that the ovary functions as a "refuge" and chemotherapeutic drugs cannot penetrate[$[9,10]$. In view of the limited effect of chemo on ovarian metastasis from CRC, local surgical resection is usually considered. Nonetheless, most previous studies have defects, including that some were case reports and case series, some had limited amount of patients, while others had no equilibrium evaluation on observation and control groups $[8,11-13]$. So, the benefit of ovariectomy has not been clearly clarified resulting in difficulties for clinicians to choose. This study was aimed to compare the survival in patients with ovariectomy plus chemo compared with those with chemo alone, and to identify the OS related prognostic factors. 
In agreement with previous studies[8], our study showed that the median OS of patients with ovarian metastasis from CRC was 19 months. We found that ovariectomy plus chemo improved survival compared with chemo alone. In the modern era of chemo, this discovery consists with historical other researches that show encouraging benefit for OS in ovariectomized patients[13, 14]. Encouragingly, when stratified analysis of the role of surgery in different time phases of ovarian metastasis, the results showed that, in metachronous metastasis but not synchronous metastasis subjects, the survival time for ovariectomy plus chemo was significantly superior to chemo alone (Figure C-D). To some extent, our results suggest that resection of metastatic tumors combined with chemo should be recommended in patients of CRC with metachronous ovarian metastasis.

It has been assumed that the ovary is a refuge for metastatic tumors, and systematic chemo is hard to penetrate into the ovarian lesions. Bevacizumab, a humanized monoclonal antibody against vascular endothelial growth factor (VEGF), has been approved by FDA as a first-line therapy for advanced CRC[15] in 2004. Mechanically, bevacizumab can change the tight junction between endothelial cells, increase vascular permeability and increase Chemo effect[16]. Whether it has a synergistic effect on ovarian metastasis from CRC remains unknown. In this study, 23(42.5\%) patients were administrated with bevacizumab treatment. In univariate survival analysis, the result indicated that the treatment of bevacizumab was involved in a significant prolongation in OS. In multivariate analysis a risk ratio of 0.65 hinted that bevacizumab treatment is beneficial but not statistically significant. This indicates that some of the survival advantages from bevacizumab treatment may be due to subjects with worse outcomes did not choose this therapy. Interestingly, the statistically significant factors were whether ovarian metastases were removed and metachronous metastasis. The level of serum CEA was marginally significant.

Considering the disastrous effects on the survival in CRC patients with ovarian metastasis, some scholars have proposed prophylactic oophorectomy. However, current studies are insufficient to identify with this hypothesis. Robin Irons and colleagues reported a retrospective case showing that prophylactic oophorectomy have possible benefits from rendering them free of suffering metsatasis[17]. Liu et al showed that CRC patients with prophylactic ovariectomy combined with N3 lymph node dissection have good oncological results[18]. While, one randomized prospective trial indicated that there was no significant survival improvment for survival rate or disease-free survival of five-years in CRC patients with prophylactic oophorectomy[19]. This treatment strategy may further require multicenter participation and a hierarchical discussion of colon and rectal cancer.

Here, we should acknowledge several limitations in this research. First, the sample size of our study is still relative small by the low incidence rate. Second, due to retrospective features, the analysis of several interested variables were limited due to deficiency of data in the clinical documentation, such as status of microsatellite instability and Kras/Nras/Braf mutations. Moreover, selection bias may exist to some extent. Since the guidelines do not specify the indications for surgery, clinically, physicians may choose the ovariectomy treatment for patients with large tumors and significant signs of compression. In addition, we conducted a landmark analysis for comparing OS between patients with or without 
bevacizumab therapy in this study, which may provide an useful information for systemic treatment. As we known, bevacizumab was permitted into the China market in 2010 and it was not covered in health insurance until 2017. Our subjects were enrolled from 2009 to 2017. So a number of patients who would benefit from bevacizumab actually did not use this drug on account of drug availability and health insurance problems. And in our used patients, we found that the majority who were administrated with bevacizumab plus chemo has the effects of partial remission or stability. Eventhough no statistically difference of OS, the hazard ratio less than 1.0 suggested that bevacizumab has potential benefits for ovarian metastasis. The effect need further big-scale investigation.

\section{Conclusion}

The current research indicated that metastasectomy plus chemo can improve the survival time of patients with ovarian metastasis from CRC, especially in metachronous metastasis subjects.

Metastasectomy and metachronous metastasis were potential prognostic factors for survival. In the future, we need to design optimized prospective studies to confirm these results, which is very important for developing the treatment strategy for ovarian metastases from CRC.

\section{Declarations}

\section{Acknowledgements}

We are grateful for all patients in the research.

\section{Authors' contributions}

TZ designed this study and critically reviewed the manuscript $\triangle M J$ and LQW had the crucial responsibility of writing the manuscript, but the other authors (LHL, DJZ, LZ, GJX, JLH) revised the manuscript for the whole content carefully.

\section{Funding}

The National Natural Science Foundation of China (No. 81602255).

\section{Availability of data and materials}

Not applicable.

\section{Ethics approval and consent to participate}

All the study participants provided their informed consent. The study design was approved by the Ethics Committee of Union Hospital affiliated to Tongji Medical College of Huazhong University of Science and Technology. 
All the authors gave approval of the final version and gave agreement for publication.

\section{Competing interests}

The authors declare that they have no competing interests.

\section{Disclosure}

All authors have declared no potential conflicts of interest.

\section{Availability of data and materials}

Not applicable

\section{References}

1. Garrett CR, George B, Viswanathan C, Bhadkamkar NA, Wen S, Baladandayuthapani V, et al. Survival benefit associated with surgical oophorectomy in patients with colorectal cancer metastatic to the ovary. Clin Colorectal Cancer. 2012; 11: 191-4.

2. Cutait R, Lesser ML, Enker WE. Prophylactic oophorectomy in surgery for large-bowel cancer. Dis Colon Rectum. 1983; 26: 6-11.

3. Birnkrant A, Sampson J, Sugarbaker PH. Ovarian metastasis from colorectal cancer. Dis Colon Rectum. 1986; 29: 767-71.

4. Hanna NN, Cohen AM. Ovarian neoplasms in patients with colorectal cancer: understanding the role of prophylactic oophorectomy. Clin Colorectal Cancer. 2004; 3: 215-22.

5. Cai J, Wang L. [Looking back 2018--focused on colorectal cancer]. Zhonghua Wei Chang Wai Ke Za Zhi. 2019; 22: 9-16.

6. Dekker E, Tanis PJ, Vleugels JLA, Kasi PM, Wallace MB. Colorectal cancer. Lancet. 2019; 394: 146780.

7. Rayson D, Bouttell E, Whiston F, Stitt L. Outcome after ovarian/adnexal metastectomy in metastatic colorectal carcinoma. J Surg Oncol. 2000; 75: 186-92.

8. Chung TS, Chang HJ, Jung KH, Park SY, Lim SB, Choi HS, et al. Role of surgery in the treatment of ovarian metastases from colorectal cancer. J Surg Oncol. 2009; 100: 570-4.

9. Goere D, Daveau C, Elias D, Boige V, Tomasic G, Bonnet S, et al. The differential response to chemotherapy of ovarian metastases from colorectal carcinoma. Eur J Surg Oncol. 2008; 34: 1335-9.

10. Taylor AE, Nicolson VM, Cunningham D. Ovarian metastases from primary gastrointestinal malignancies: the Royal Marsden Hospital experience and implications for adjuvant treatment. $\mathrm{Br} \mathrm{J}$ Cancer. 1995; 71: 92-6.

11. Ojo J, De Silva S, Han E, Lin P, Wakabayashi M, Nelson R, et al. Krukenberg tumors from colorectal cancer: presentation, treatment and outcomes. Am Surg. 2011; 77: 1381-5. 
12. Jiang R, Tang J, Cheng X, Zang RY. Surgical treatment for patients with different origins of Krukenberg tumors: outcomes and prognostic factors. Eur J Surg Oncol. 2009; 35: 92-7.

13. Erroi F, Scarpa M, Angriman I, Cecchetto A, Pasetto L, Mollica E, et al. Ovarian metastasis from colorectal cancer: prognostic value of radical oophorectomy. J Surg Oncol. 2007; 96: 113-7.

14. Fujiwara A, Noura S, Ohue M, Shingai T, Yamada T, Miyashiro I, et al. Significance of the resection of ovarian metastasis from colorectal cancers. J Surg Oncol. 2010; 102: 582-7.

15. Hurwitz H, Fehrenbacher L, Novotny W, Cartwright T, Hainsworth J, Heim W, et al. Bevacizumab plus irinotecan, fluorouracil, and leucovorin for metastatic colorectal cancer. N Engl J Med. 2004; 350: 2335-42.

16. Cohen AF, van Bronswijk H. [New medications; bevacizumab]. Ned Tijdschr Geneeskd. 2006; 150 : 2194-5.

17. Irons R, Mclntosh E, Hageboutros A, Warshal D, McClane S. Bilateral ovarian micrometastatic adenocarcinoma upon prophylactic oophorectomy concurrent with low anterior resection for rectal cancer. World J Surg Oncol. 2017; 15: 40.

18. Liang JT, Lai HS, Wu CT, Huang KC, Lee PH, Shun CT. Laparoscopic prophylactic oophorectomy plus N3 lymphadenectomy for advanced rectosigmoid cancer. Ann Surg Oncol. 2007; 14: 1991-9.

19. Young-Fadok TM, Wolff BG, Nivatvongs S, Metzger PP, Ilstrup DM. Prophylactic oophorectomy in colorectal carcinoma: preliminary results of a randomized, prospective trial. Dis Colon Rectum. 1998; 41: 277-83; discussion 83-5.

\section{Tables}


Table 1: Clinical characteristics

$$
\begin{array}{llll}
\begin{array}{l}
\text { Ovariectomy plus chemo }(\mathrm{n}=34), \\
\mathrm{n}(\%)
\end{array} & \begin{array}{l}
\text { Chemo } \\
\text { alone }
\end{array} & \begin{array}{l}
\text { Total } \\
(\mathrm{n}=54),
\end{array} & \begin{array}{l}
\mathrm{P} \\
\text { value }
\end{array} \\
& \begin{array}{l}
(\mathrm{n}=20), \mathrm{n} \\
(\%)
\end{array} & \mathrm{n}(\%) & \\
& &
\end{array}
$$

\begin{tabular}{|c|c|c|c|}
\hline Stage $\otimes / \mathbb{}$ & $12(35)$ & 7 (35) & $19(35)$ \\
\hline Stage $\rrbracket$ & $22(65)$ & $13(65)$ & $35(65)$ \\
\hline
\end{tabular}

\begin{tabular}{lllll} 
Age,median(range),y & $44.0(17-75)$ & $47.5(20-$ & $45.3(17-$ & 0.31 \\
\hline Tumor Size & $10.5 \pm 5.7$ & $67)$ & $75)$ & \\
(mean,cm) & & $8.5 \pm 6.4$ & $10.0 \pm 5.9$ & 0.08 \\
\hline
\end{tabular}

\section{Tumor staging}

\section{Pathology}

$\begin{array}{lllll}\text { Adenocarcinoma } & 25(94) & 15(97) & 51(81) & 0.90 \\ \text { Others } & 2(6) & 1(3) & 3(9) & \end{array}$

\section{Differentiation}

\begin{tabular}{lllll} 
Poorly & $22(65)$ & $13(65)$ & $35(65)$ & 0.98 \\
Well and moderately & $12(35)$ & $7(35)$ & $19(35)$ & \\
Laterality & & & & \\
\hline Unilateral & $20(59)$ & $9(45)$ & $29(54)$ & 0.32 \\
\hline Bilateral & $14(41)$ & $11(55)$ & $25(46)$ & \\
\hline Chronology & & & & \\
\hline synchronous & $15(44)$ & $8(40)$ & $23(43)$ & 0.77 \\
metachronous & $19(56)$ & $12(60)$ & $31(57)$ & \\
\hline Primary site & & & & \\
\hline colon & $30(88)$ & $20(100)$ & $50(93)$ & $4(7)$
\end{tabular}

\section{Extent of metastasis}

Only ovarian metastasis $6(18)$

Beyond ovarian 28 (82) metastasis

$$
28 \text { (82) }
$$

$11(20 \%) \quad 0.32$

$15(75 \%) \quad 43(80 \%)$




\section{Peritoneum metastasis}

$\begin{array}{lllll}\text { Yes } & 18(53) & 8(40) & 26(48) & 0.35 \\ \text { No } & 16(47) & 12(60) & 28(52) & \end{array}$

\section{Liver metastasis}

$\begin{array}{lllll}\text { Yes } & 9(26) & 7(35) & 16(30) & 0.51 \\ \text { No } & 25(74) & 13(65) & 38(70) & \end{array}$

\section{Lung metastasis}

Yes

$10(29)$

$5(25)$

15 (28)

0.73

No

$24(71)$

$15(75)$

39 (72)

\section{Bevacizumab treatment}

$\begin{array}{lllll}\text { Yes } & 10(29) & 3(15) & 13(24) & 0.39 \\ \text { No } & 24(71) & 17(85) & 41(76) & \end{array}$

\section{Serum CA125(U/mL)}

$\begin{array}{lllll}>37 & 10(29) & 9(45) & 19(35) & 0.25 \\ \leq 37 & 24(71) & 11(55) & 35(65)\end{array}$

\section{Serum CEA}

$(\mathrm{U} / \mathrm{mL})$

$\begin{array}{lllll}>5 & 21(62) & 11(55) & 32(59) & 0.78 \\ \leq 5 & 13(38) & 9(45) & 22(41) & \end{array}$

\section{Serum CA199 (U/mL)}

$\begin{array}{lllll}>35 & 12(35) & 8(40) & 20(37) & 0.73 \\ \leq 35 & 22(65) & 12(60) & 34(63)\end{array}$

NLR

$\begin{array}{lllll}>2.08 & 20(59) & 8(40) & 28(52) & 0.26 \\ \leq 2.08 & 14(41) & 12(60) & 26(48) & \\ \text { NPR } & & & & \\ >137 & 18(53) & 10(50) & 28(52) & 0.83 \\ \leq 137 & 16(47) & 10(50) & 26(48) & \end{array}$


Table 2: Univariate and multivariate analysis for ovarial survival

\begin{tabular}{|c|c|c|c|c|}
\hline \multirow[t]{2}{*}{ Statistical variables } & \multicolumn{2}{|l|}{ Univariate analysis } & \multicolumn{2}{|c|}{ Multivariate analysis } \\
\hline & $\mathrm{HR}(95 \% \mathrm{Cl})$ & P Value & $\mathrm{HR}(95 \% \mathrm{Cl})$ & P Value \\
\hline Age $₫ 40 y$ & $0.70(0.36-1.37)$ & 0.28 & - & - \\
\hline Colon cancer & $1.02(0.82-1.26)$ & 0.83 & - & - \\
\hline Adenocarcinoma & $0.66(0.69-1.26)$ & 0.52 & - & - \\
\hline Poorly differentiation & $1.72(0.85-3.48)$ & 0.13 & - & - \\
\hline 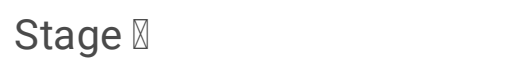 & $2.29(1.14-4.61)$ & 0.02 & $1.76(0.78-3.99)$ & 0.17 \\
\hline Metachronous metastases & $0.39(0.20-0.70)$ & {$[0.01$} & $0.46(0.22-0.95)$ & 0.03 \\
\hline Bilateral metastases & $1.06(0.59-1.89)$ & 0.83 & - & - \\
\hline Extraovarian metastases & $1.31(0.62-2.73)$ & 0.47 & - & - \\
\hline Peritoneum metastases & $1.43(0.80-2.55)$ & 0.23 & - & - \\
\hline Liver metastases & $2.86(1.47-5.54)$ & $\square 0.01$ & $1.62(0.71-3.62)$ & 0.23 \\
\hline Lung metastases & $2.11(1.09-4.06)$ & 0.02 & $1.94(0.84-4.47)$ & 0.12 \\
\hline ovariectomy plus chemo & $0.41(0.22-0.77)$ & 00.01 & $0.40(0.20-0.79)$ & 00.01 \\
\hline Bevacizumab treatment & $0.47(0.24-0.93)$ & 0.02 & $0.64(0.30-1.37)$ & 0.26 \\
\hline CA125 >37 U/mL & $2.04(1.11-3.77)$ & 0.03 & $1.39(0.64-3.01)$ & 0.40 \\
\hline $\mathrm{CEA}>5 \mathrm{U} / \mathrm{mL}$ & $1.99(1.05-3.76)$ & 0.03 & $2.04(0.90-4.59)$ & 0.08 \\
\hline CA199 >35 U/mL & $1.48(0.82-2.70)$ & 0.13 & - & - \\
\hline NLR $>2.08$ & $1.31(0.73-2.37)$ & 0.36 & - & - \\
\hline PLR >137 & $1.43(0.78-2.63)$ & 0.22 & - & - \\
\hline
\end{tabular}

NLR: neutrophil-to-lymphocyte ratio; PLR: platelet-to-lymphocyte ratio

\section{Figures}



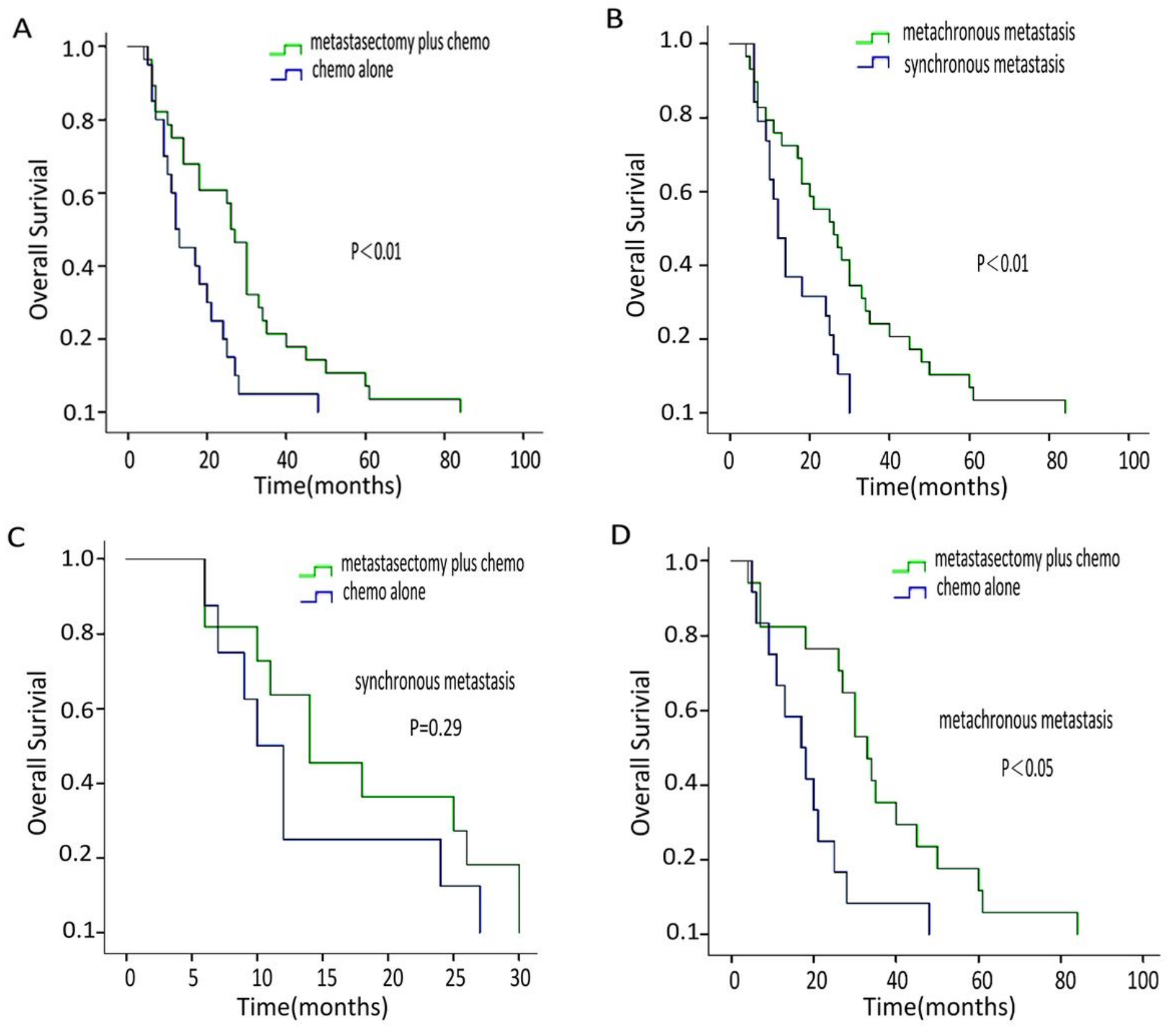

Figure 1

Kaplan-Meier analysis of Overall Survival. A Survival of metastasectomy plus chemo vs. chemo ; B Survival of synchronously metastasis vs. metachronous metastasis, C Survival of metastasectomy plus chemo vs. chemo in patients with synchronously metastasis, D Survival of metastasectomy plus chemo vs. chemo in patients with metachronous metastasis 